\title{
Difference Frequency of Kilohertz QPOs Not Equal to Half the Burst Oscillation Frequency in 4U 1636-53
}

\author{
Mariano Méndez ${ }^{1,2}$, Michiel van der Klis ${ }^{1}$, Jan van Paradijs ${ }^{1,3}$,
}

\begin{abstract}
We have analyzed data obtained during two observations with the Rossi X-ray Timing Explorer on January 5 and 8, 1997, of the low-mass X-ray binary (LMXB) and atoll source $4 \mathrm{U} 1636-53$. We measure the frequency separation of the two simultaneous kilohertz quasi-periodic oscillations (kHz QPOs) in this source to be $253.7 \pm 4.7$ and $246.4 \pm 5.4 \mathrm{~Hz}$, respectively. These values are inconsistent with being equal to 0.5 times the frequency of the $581-\mathrm{Hz}$ oscillations that have been detected previously in $4 \mathrm{U} 1636-$ 53 during type I bursts. The weighted average discrepancy is $39.5 \pm 3.5 \mathrm{~Hz}$. This result shows that a simple beat-frequency interpretation of the $\mathrm{kHz}$ QPOs, in which the frequency of the oscillations detected during type I bursts equals the separation between the two $\mathrm{kHz}$ QPOs (or twice that value), is incorrect.
\end{abstract}

Subject headings: accretion, accretion disks — stars: neutron — stars: individual (4U 1636-53) - X-rays: stars

\footnotetext{
${ }^{1}$ Astronomical Institute "Anton Pannekoek", University of Amsterdam and Center for High-Energy Astrophysics, Kruislaan 403, NL1098 SJ Amsterdam, the Netherlands

${ }^{2}$ Facultad de Ciencias Astronómicas y Geofísicas, Universidad Nacional de La Plata, Paseo del Bosque S/N, 1900 La Plata, Argentina

${ }^{3}$ Physics Department, University of Alabama in Huntsville, Huntsville, AL 35899, USA
} 


\section{Introduction}

Observations with the Rossi X-ray Timing Explorer (RXTE) have so far revealed coherent oscillations during type I X-ray bursts in 6 low-mass Xray binaries (LMXBs): in $4 \mathrm{U} 1728-34$ at $363 \mathrm{~Hz}$ (Strohmayer et al. 1996a, 1997b), in 4U 1636-53 at $581 \mathrm{~Hz}$ (Zhang et al. 1996, Strohmayer et al. 1998), in KS 1731-26 at $524 \mathrm{~Hz}$ (Smith et al. 1997), in $4 \mathrm{U}$ $1702-43$ at $330 \mathrm{~Hz}$ (Strohmayer, Swank and Zhang 1998), in Aql X-1 at $549 \mathrm{~Hz}$ (Zhang et al. 1998), and in a source near the Galactic Center (probably MXB 1743-29) at $589 \mathrm{~Hz}$ (Strohmayer et al. 1997a). The first four sources have also shown two simultaneous kilohertz quasi-periodic oscillations ( $\mathrm{kHz}$ QPOs) in their persistent emission (Strohmayer et al. 1996a; Wijnands et al. 1997; Wijnands \& van der Klis 1997; Swank 1998). In 4U 1728-34 the separation between the two simultaneous $\mathrm{kHz}$ QPOs was $355 \pm 5 \mathrm{~Hz}$, consistent with the frequency of the oscillations during the type I bursts. In $4 \mathrm{U} 1702-43$ the $\mathrm{kHz}$ QPO separation frequency and the burst oscillation frequency were also similar. In 4U 1636-53 and KS 1731-26 the frequency separation of the $\mathrm{kHz}$ peaks was $276 \pm 10$ $\mathrm{Hz}$ and $260 \pm 10 \mathrm{~Hz}$, respectively, consistent with half the frequency during the burst oscillations.

These results strongly suggested that the burst oscillations and the twin $\mathrm{kHz}$ QPO are connected through a beat frequency relation, in which signals at two of the frequencies are interacting to produce a third one at their difference frequency. There are good arguments that the burst oscillations are due to shortlived thermonuclear-powered hot spots on the neutron star surface that spin around with approximately the star's spin frequency (Strohmayer et al. 1998). In a beat frequency interpretation a natural choice for the two interacting oscillations then is (i) the burst oscillations, occurring at the neutron star spin frequency (or twice that), and the (ii) the higher-frequency $\mathrm{kHz}$ QPO, occurring at the Kepler frequency corresponding to some preferred radius in the accretion disk (Strohmayer et al. 1996b; Miller, Lamb, \& Psaltis 1998). The lower-frequency $\mathrm{kHz}$ QPO is then due to the beat between these two frequencies. In such an interpretation the $\mathrm{kHz}$ QPO frequency difference is equal to the neutron star spin frequency and hence predicted to be constant. In most of the sources that showed the simultaneous $\mathrm{kHz}$ QPO peaks the frequency difference was indeed consistent with being constant as the twin $\mathrm{kHz}$ peaks moved up and down in frequency as a function of, presumably, accretion rate.

In sources for which only the twin $\mathrm{kHz} \mathrm{QPO}$, and no burst oscillations, were observed the frequency difference was interpreted in terms of the neutron star spin frequency as well. However, we now know that two of these sources do not fit this interpretation: both in Sco X-1 (van der Klis et al. 1997), and in 4U 1608-52 (Méndez et al. 1998b) the separation between the two simultaneous $\mathrm{kHz}$ QPOs varies significantly, by $\sim 40 \%$ on time scales of a few days, showing that at least in these sources the separation frequency can not be simply the spin frequency.

In this Letter we present new results that conclusively show that the frequency separation of the $\mathrm{kHz}$ QPOs in 4U 1636-53 differs from being equal to half the frequency of the oscillations detected during type I X-ray bursts in this source. In Section 2 we describe the observations and data analysis. In Section 3 we discuss our findings.

\section{Observations and Data Analysis}

We have analyzed two observations of $4 \mathrm{U} 1636$ 53 carried out with the Proportional Counter Array (PCA) on board RXTE during January 1997. The first started on 1997 January 5, 22:05 UTC and lasted for $\sim 19.5 \mathrm{ks}$, and the second one started on January 8, 04:33 UTC and lasted $\sim 12.5 \mathrm{ks}$. In both observations data were collected using an Event mode with $1 / 8192$ s time resolution and 64 energy channels covering the nominal 2-60 keV energy band of the PCA. No type I X-ray bursts were detected.

We divided these high-time resolution $2-60 \mathrm{keV}$ data into segments of $64 \mathrm{~s}$, Fourier transformed these, and for each segment produced a power spectrum extending from $1 / 64$ to $2048 \mathrm{~Hz}$. In both observations a strong $\mathrm{kHz}$ QPO peak was visible. The centroid frequency of this peak varied between $830 \mathrm{~Hz}$ and 930 $\mathrm{Hz}$ on January 5, and between $890 \mathrm{~Hz}$ and $1050 \mathrm{~Hz}$ on January 8. In both cases, a second, less significant peak was detected at a frequency 200 to $300 \mathrm{~Hz}$ higher than that.

We repeated the procedure using data in several different energy bands. While the lower-frequency QPO was significant in all of these bands, the higherfrequency QPO was more significant when we only used data above $5 \mathrm{keV}$. For this reason, and to reduce the influence of the background at high energies, we decided to use only the data between 5 and 
$20 \mathrm{keV}$. Again, we produced a power spectrum every $64 \mathrm{~s}$. As the lower-frequency QPO was well detected in each segment, we fitted its central frequency and then shifted the frequency scale of each spectrum to a frame of reference where the position of the most significant peak was constant in time (see Méndez et al. 1998a . Finally, we averaged these shifted spectra to produce one single 5-20 keV power spectrum per observation.

We fitted the 256-1500 Hz frequency range of each of these two average power spectra using a function consisting of a constant, representing the Poisson noise, and two Lorentzians, representing the QPOs. The fits were good, with reduced $\chi^{2} \leq 1.1$. We show in Figure 1 the average power spectrum of the two observations and the model that we used to fit it. The 5-20 keV amplitudes and FWHM of the lower frequency QPO were 8.6 $\pm 0.1 \% \mathrm{rms}$ and $13.2 \pm 0.4 \mathrm{~Hz}$, respectively on January 5 , and $3.5 \pm 0.2 \% \mathrm{rms}$ and $6.2 \pm 0.7 \mathrm{~Hz}$, respectively on January 8. The amplitudes and FWHM of the higher frequency QPO were $5.2 \pm 0.4 \% \mathrm{rms}$ and $70 \pm 12 \mathrm{~Hz}$, and $4.8 \pm 0.4 \% \mathrm{rms}$ and $64 \pm 14 \mathrm{~Hz}$, respectively. The frequency difference between the two simultaneous $\mathrm{kHz}$ QPOs was consistent with being constant. The separation between the two peaks was $253.7 \pm 4.7 \mathrm{~Hz}$ on January 5 , and $246.4 \pm 5.4$ on January 8 . The average separation during these two observations as measured from the overall average power spectrum was the same as the weighted average of these two values: $250.6 \pm 3.5 \mathrm{~Hz}$.

\section{Discussion}

Our measured average $\mathrm{kHz}$ QPO peak separation, $250.6 \pm 3.5 \mathrm{~Hz}$, is inconsistent with being equal to half the burst oscillation frequency at better than $10 \sigma$ confidence. Although the burst oscillation frequency shows a small $(<2 \mathrm{~Hz})$ drift during some bursts, it is statistically very well determined and seems to always be between 579 and $582 \mathrm{~Hz}$ (see Strohmayer, Swank and Zhang 1998, and Strohmayer et al. 1998). So, the separation frequency is too small by between $38.9 \pm 3.5$ and $40.4 \pm 3.5 \mathrm{~Hz}$ to be equal to half the burst oscillation frequency.

In the beat frequency model for the $\mathrm{kHz}$ QPOs (Section 1), the two basic frequencies of the system are the Keplerian frequency of the accreting material in orbit around the neutron star at some preferred radius, identified with the high frequency $\mathrm{kHz} \mathrm{QPO}$, at a frequency $\nu_{\mathrm{upp}}$, and the spin frequency of the neutron star, $\nu_{\mathrm{s}}$. These two frequencies beat with each other to produce the low-frequency $\mathrm{kHz}$ QPO at $\nu_{\text {low }}=\nu_{\text {upp }}-\nu_{\mathrm{s}}$. So, the model predicts that $\Delta \nu \equiv$ $\nu_{\text {upp }}-\nu_{\text {low }}=\nu_{s}$.

As noted in Section 1, this model agrees well with the results obtained in 4U 1728-34 (Strohmayer et al. 1996a) if one assumes that $\nu_{\mathrm{s}}$ in this source is equal to the frequency $\nu_{\text {burst }}$ of the oscillations observed during bursts. In $4 \mathrm{U} 1636-53$ and KS $1731-26, \Delta \nu$ is approximately equal to $\nu_{\text {burst }} / 2$ (Wijnands et al. 1997; Wijnands \& van der Klis 1997). A beat-frequency model could perhaps account for this if in these two sources $\nu_{\mathrm{s}}=\nu_{\text {burst }} / 2$. This could occur if, for example, two hot spots are present during the burst. (This interpretation has been contested by Strohmayer et al. 1998 on the basis of the large observed amplitudes of the oscillations.)

Our results show conclusively that, at least in $4 \mathrm{U}$ 1636-53, this simple beat frequency picture is invalid. If we assume that $\nu_{\mathrm{S}}=\nu_{\text {burst }} / 2$, then $\nu_{\mathrm{s}}$ exceeds $\Delta \nu$ by a value near $40 \pm 3.5 \mathrm{~Hz}$, a discrepancy of more than $10 \sigma$. (Of course, the situation would be worse if $\nu_{\mathrm{s}}=$ $\left.\nu_{\text {burst }}=581 \mathrm{~Hz}\right)$.

The beat-frequency model for the twin $\mathrm{kHz} \mathrm{QPO}$ in LMXBs has been strongly challenged by the measurements of a variable peak separation in Sco X-1 and 4U 1608-52 (van der Klis et al. 1997; Méndez et al. 1998b). Our findings show that the beat frequency model in its current form fails in the case of $4 \mathrm{U} 1636$ 53. This is the first time that this is demonstrated in an object where all three of the frequencies involved in the beat-frequency interpretation are measurable. In view of the great similarity of the $\mathrm{kHz}$ QPO phenomenology in all LMXBs this conclusion also applies to the phenomenon as observed in other LMXBs.

It may be too early to discard the beat frequency model altogether. It is conceivable that the $\mathrm{kHz}$ peak separation frequency, while not being equal to the neutron star spin frequency (or twice that) is still constrained to be relatively near it. This could be the case if it is the spin frequency of accreting matter that is not corotating with the star. It is also possible that the beat frequency is generated by processes occurring at a radius that differs from the radius inferred from the Kepler frequency interpretation of the upper $\mathrm{kHz}$ QPO frequency. In view of the complexity apparently involved in a successful beat frequency model for the $\mathrm{kHz} \mathrm{QPO}$, in our opinion constraints on physical parameters such as neutron star spin frequency, mass, and radius inferred from the properties 
of these QPO, must for the time being be interpreted with great care.

This work was supported in part by the Netherlands Organization for Scientific Research (NWO) under grant PGS 78-277 and by the Netherlands Foundation for research in astronomy (ASTRON) under grant 781-76-017. MM is a fellow of the Consejo Nacional de Investigaciones Científicas y Técnicas de la República Argentina. JVP acknowledges support from the National Aeronautics and Space Administration through contract NAG 5-3269 and 5-4482.

\section{REFERENCES}

Méndez, M., et al. 1998a, ApJ, 494, L65

Méndez, M., van der Klis, M., Wijnands, R., Ford, E. C., van Paradijs, J., \& Vaughan, B. A. 1998b, ApJ, 505, L23

Miller, M. C., Lamb, F. K., Psaltis, D. 1998, ApJ, in press astro-ph/9609157)

Smith, D. A., Morgan, E. H., \& Bradt, H. 1997, ApJ, 479,L137

Strohmayer, T. E., Jahoda, K., Giles, B., \& Lee, U. 1997a, ApJ, 486, 355

Strohmayer, T. E., Zhang, W., Smale, A., Day, C., Swank, J. H., Titarchuk, L., \& Lee, U. 1996b, IAU Circ. 6387

Strohmayer, T. E., Zhang, W., \& Swank, J. H. 1997b, ApJ, 487, L77

Strohmayer, T. E., Zhang, W., Swank, J. H., Smale, I., Titarchuk, L., Day, C. \& Lee, U. 1996a, ApJ, 469, L9

Strohmayer, T. E., Zhang, W., \& Swank, J. H., White, N. E., Lapidus, I. 1998, ApJ, 498, L135

Strohmayer, T. E., Zhang, W., \& Swank, J. H., 1998, Proceedings of the Symposium "The Active X-Ray Sky: Results from BeppoSAX and RossiXTE", Rome, Italy, 21-24 October, 1997, Eds. L. Scarsi, H. Bradt, P. Giommi, and F. Fiore, Nuclear Physics B Proceedings Supplements. 1998, in press astro-ph/9801219)

Swank, J.H., Proceedings of IAU Symposium 188: The Hot Universe, in press (astro-ph/9801218) van der Klis, M., Wijnands, R. A. D., Horne, K., \& Chen, W. 1997, ApJ, 481, L97

Wijnands, R. A. D., \& van der Klis, M. 1997, ApJ, 482, L65

Wijnands, R. A. D., van der Klis, M., van Paradijs, J., Lewin, W. H. G., Lamb, F. K., Vaughan, B. A., \& Kuulkers, E. 1997 ApJ, 479, L141

Zhang, W., Lapidus, I., Swank, J. H., White, N. E., \& Titarchuk, L. 1996, IAU Circ. 6541

Zhang, W., Jahoda, K., Kelley, R. L., Strohmayer, T. E., Swank, J. H., \& Zhang, S. N. 1998, ApJ, 495, L9

This 2-column preprint was prepared with the AAS LATEX macros $\mathrm{v} 4.0$. 


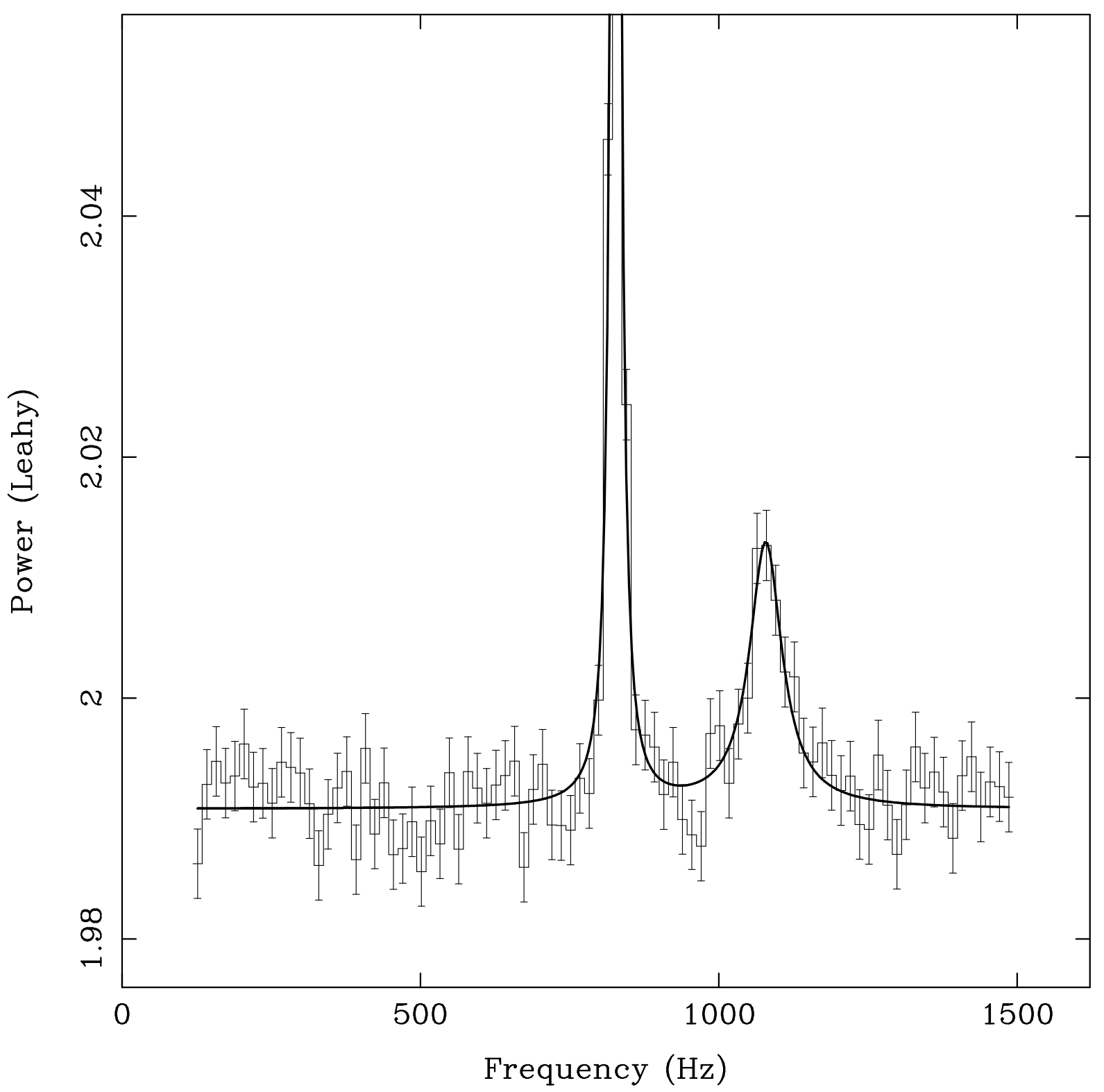

Fig. 1.- Average power spectrum of the two observations presented in this paper, in the $5-20 \mathrm{keV}$ energy range. The frequency of the lower-frequency peak as measured in 64-s data segments was arbitrarily shifted to improve the detection of the QPO at higher frequencies (see Méndez et al. 1998a for details). The solid line represents the best fit model as described in the text. The frequency separation of the two peaks is $250.6 \pm 3.5 \mathrm{~Hz}$. 